\title{
Alcohol-related brain injury: Improving identification and management in an acute care setting
}

\author{
Authors: Lynn Owens, ${ }^{A, B}$ Andrew Thompson, ${ }^{B}$ Kev Patterson ${ }^{A}$ and Paul Richardson ${ }^{A}$
}

\section{Aims}

To increase detection of alcohol-related brain injury (ARBI) in patients attending hospital with an alcohol use disorder.

\section{Methods}

In April 2017, we implemented an innovative clinical pathway. We developed an electronic warning system to identify those patients at risk of ARBI. Criteria for referral were developed utilising an algorithm based on the number of previous emergency department attendances or hospital admissions and concerns raised by relatives or staff. For patients meeting criteria, an automatic referral was made to a specialist nurse for assessment utilising the Montreal Cognitive Assessment tool (MoCA). A score of $<23$ was considered positive for potential ARBI. This triggered a referral to a psychiatrist for confirmation of diagnosis. We performed a 6 -month follow-up descriptive evaluation.

\section{Results}

Of 193 patients screened using our algorithm, 69 (35.7\%) screened positive for ARBI utilising criteria of MoCA $<23$ (confirmed by psychiatric assessment). Compared to the number of patients identified in the 6 months prior to the introduction of our pathway, identification of ARBI was increased from an average of $\sim 2$ per month to an average of $\sim 8$.

Improved engagement in alcohol treatment with retention rates at 3 months of $70 \%$.

Reduction in alcohol consumption with $32 \%$ remaining totally abstinent and a reduction from an average of 25 units per day pre-intervention to 16 units per day post-intervention for those who continued to drink.

Average $50 \%$ reduction in acute hospital utilisation 3 month pre- and post-detection (3.2 compared to 1.5 for attendance and 1.5 compared to 0.6 for admission) this resulted in a saving of $\sim £ 63,600$ based on analysis of healthcare resource groups costings.

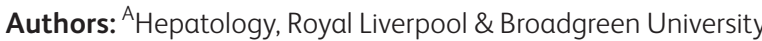
Hospital, Liverpool, UK; ${ }^{B}$ Molecular and clinical pharmacology, Translational Medicine, Liverpool, UK
Results from family-reported outcome measures has highlighted several outcomes that our patient families found most valuable: receiving an assessment to confirm or reject the presence of ARBI, helping them understand their loved ones condition and helping them plan for the future.

\section{Conclusion}

We have demonstrated MoCA to be an effective clinical tool for determining ARBI, as well as being both feasible and acceptable. Therefore, we have been able to embed MoCA screening into usual clinical care for alcohol specialist nurse interventions. This point-of-care screening can facilitate the initiation of appropriate referral and treatment pathways which ultimately improve patients' outcomes. Patients and their families reported previous feeling of shame due to perceived poor compliance with treatment to finally being listened to and treated with respect and dignity.

\section{Conflict of interest statement}

None declared. 